\title{
Geometric and electronic structures of one-dimensionally polymerized coronene molecules
}

\author{
Kohei Narita* and Susumu Okada \\ Graduate School of Pure and Applied Sciences, University of Tsukuba, Tsukuba, Ibaraki 305-8571, Japan
}

\begin{abstract}
On the basis of density functional theory, we studied the energetics, geometries, and electronic structures of dicoronylene molecules (coronene dimers) and one-dimensionally polymerized coronene molecules. Our calculations showed that the relative stability of the coronene dimers and polymers strongly depends on the mutual arrangement of molecules with respect to the polymer or dimer directions: the coronene dimer and the polymer with six-membered rings in the intermolecular bonds are the most stable structures among the three possible intermolecular arrangements. The dimer possesses a large energy gap between the highest occupied and lowest unoccupied states, which is narrower than that of the isolated coronene. The polymers are semiconductors with a moderate direct band gap and a large indirect gap for the polymers with six-membered and four-membered rings and that with five-membered rings, respectively, indicating the possibility of the electronic structure control of polymers via the intermolecular arrangement.
\end{abstract}

\section{Introduction}

In this decade, graphene has been attracting a great deal of attention due to its unusual electronic and geometric structures, ${ }^{1-8)}$ which suggest its possible use as a constituent material for electronic, energy, sensing, and spintronic devices in the near future. ${ }^{9-14)}$ For the practical application of graphene in such devices, it is mandatory to precisely control the atomic structure of graphene flakes due to the diversity of their electronic structure, which sensitively depends on their size and shape. ${ }^{15,16)}$ The cyclodehydrogenation of polycyclic aromatic hydrocarbon (PAH) molecules is one possible procedure for synthesizing structurally well-controlled graphene flakes that are applicable to a wide range of modern technologies. ${ }^{17,18)}$ The most important advantage of this synthetic technique is that the size and shape of the resultant graphitic nanostructures are, in principle, atomistically controllable by assembling appropriate PAH molecules under optimum external conditions. For instance, the thermally activated cyclodehydrogenation of PAH molecules on metal surfaces leads to nanoscale graphene flakes whose size and dimen-

\footnotetext{
*E-mail: knarita@comas.frsc.tsukuba.ac.jp
} 
sions are determined by the molecular shape and size of the precursor. In addition, the origomerization and polymerization of coronene molecules $\left(\mathrm{C}_{24} \mathrm{H}_{12}\right)^{19,20)}$ inside carbon nanotubes has been experimentally demonstrated. The resulting structures can be regarded as a prototype of narrow graphene nanoribbons with edge roughness. ${ }^{21,22)}$ Despite intensive experimental works on one-dimensional chains of polymeric coronene, detailed geometric and electronic structures of the polymeric phases have not yet been determined, because there are several possible intermolecular arrangements, which sensitively affect the physical properties of these coronene polymers.

In this work, we study the geometric and electronic structures of dicoronylene molecules and one-dimensionally polymerized coronene molecules based on firstprinciples total-energy calculations to obtain a theoretical insight into the fundamental properties of ultranarrow graphene nanoribbons with edge roughness. Our calculations show that two of the three dicoronylenes studied here have relatively small total energy. The electronic structures of these dicoronylenes have a wide energy gap between the highest occupied and lowest unoccupied states, similarly to a coronene monomer. By connecting these dicoronylenes by cyclodehydrogenation, we consider three structures of one-dimensionally polymerized coronene. These three polymeric coronene chains are semiconductors with direct and indirect energy gaps depending on the mutual intermolecular arrangement. We also demonstrated that the polymers possess a moderate Young's modulus of 530-620 GPa. The results indicate that coronene and the dicoronylenes can be constituent units for narrow semiconducting wires with moderate mechanical strength, whose electronic structures are tunable by controlling the intermolecular cyclodehydrogenation.

\section{Calculation methods and structural model}

All calculations were performed in the framework of density functional theory $(\mathrm{DFT})^{23,24)}$ using the Simulation Tool for Atom TEchnology (STATE) package. ${ }^{25)}$ To calculate the exchange-correlation energy among the interacting electrons, we used the generalized gradient approximation (GGA) including the spin degree of freedom with the functional forms of Perdew-Burke-Ernzerhof (PBE) to investigate the possibility of the spin polarization. ${ }^{26)}$ Ultrasoft pseudopotentials generated using the Vanderbilt scheme were employed to describe the interaction between electrons and ions. The valence wave functions and charge density were expanded in terms of the plane-wave basis set with cutoff energies of 25 and 225 Ry, respectively. Integration over the Brillouin 
(a)

Dicoronylene [6]

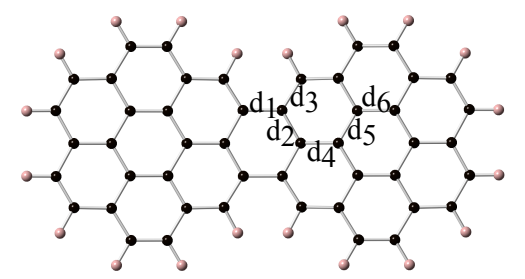

(b)

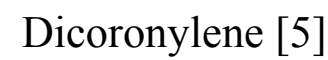

(c)

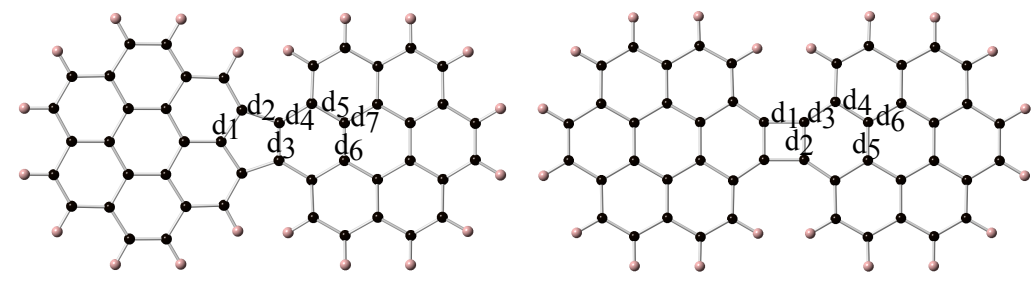

Fig. 1. Geometric structures of dicoronylene molecules in which coronene molecules are connected via (a) six-membered, (b) five-membered, and (c) four-membered rings.

zone was carried out using the $\Gamma$ point and an equidistant mesh of 4 - $\boldsymbol{k}$ points for dicoronylene molecules and coronene polymers, respectively. Structural optimization was performed until the remaining force acting on each atom was less than $5 \mathrm{mRy} / \AA$ for each lattice constant.

\section{Results and discussion}

We consider three dicoronylene molecules, which are generated by the cyclodehydrogenation of coronene molecules. Figure 1 shows the fully optimized geometries of the dicoronylene molecules. Among these three structures, two dicoronylenes have been experimentally synthesized, ${ }^{27)}$ in which coronene molecules are connected via hexagonal [Fig. 1(a)] and pentagonal [Fig. 1(b)] rings by dehydrogenation. In addition, we consider another possible dicoronylene that contains a four-membered ring in the intermolecular covalent bonds [Fig. 1(c)]. As shown in Fig. 1, each coronene unit retains its monomer shape. Indeed, as summarized in Table I, the optimized bond lengths of the dicoronylenes are 1.38-1.43 $\AA$ within the coronene unit, which are almost identical to those in a coronene monomer. For the intermolecular bonds, the optimized lengths are $1.46,1.47$, and $1.49 \AA$ for six-membered, five-membered, and four-membered rings, respectively.

Table I. Bond lengths of dicoronylene molecules (in $\AA$ ). Bond indexes are listed in Fig. 1.

\begin{tabular}{cccccccc}
\hline & $\mathrm{d} 1$ & $\mathrm{~d} 2$ & $\mathrm{~d} 3$ & $\mathrm{~d} 4$ & $\mathrm{~d} 5$ & $\mathrm{~d} 6$ & $\mathrm{~d} 7$ \\
\hline Dicoronylene [6] & 1.46 & 1.42 & 1.38 & 1.42 & 1.41 & 1.41 & \\
Dicoronylene [5] & 1.41 & 1.47 & 1.41 & 1.41 & 1.42 & 1.42 & 1.42 \\
Dicoronylene [4] & 1.49 & 1.39 & 1.38 & 1.43 & 1.42 & 1.41 & \\
\hline
\end{tabular}


Table II. Relative total energies of dicoronylene molecules. Energies are measured from that of the ground state polymer containing six-membered rings between coronene molecules.

\begin{tabular}{lc}
\hline & Energy $(\mathrm{eV} /$ coronene $)$ \\
\hline Dicoronylene [6] & 0 \\
Dicoronylene [5] & 0.21 \\
Dicoronylene [4] & 1.18 \\
\hline
\end{tabular}

The total energies of the dicoronylenes are listed in Table II. The isomer containing a six-membered ring between molecules is the most stable among the three isomers studied here. On the other hand, the isomer with the four-membered ring is the least stable. The relative stability of the isomer with a five-membered ring with respect to that of the isomer with a six-membered ring is the same as that of the $\pi$ electron energy evaluated in an early Huckel calculation. ${ }^{28)}$ The relative stability of the three isomers is attributed to the distortion of intermolecular bonds from the ideal $\mathrm{sp}^{2}$ covalent bond. As shown in Fig. 1 and listed in Table I, the intermolecular bonds of the four-membered and five-membered rings are slightly elongated compared with those of the hexagonal ring. Furthermore, the four-membered ring considerably increases the total energy of the isomer compared with those of the other two isomers due to the highly distorted intermolecular bonds.

Figure 2 shows the electronic structures of the dicoronylenes. The calculated energy gaps between the highest occupied (HO) and lowest unoccupied (LU) Kohn-Sham states are $1.40,1.99$, and $1.78 \mathrm{eV}$ for the isomers with the six-membered, five-membered, and four-membered rings, respectively. The HO-LU gap of an isolated coronene molecule is $2.95 \mathrm{eV}$ in DFT with the local density approximation; thus, the dimerization causes a decrease in the HO-LU gap. Among the three isomers, the isomer with the fourmembered ring possesses a relatively shallow HO state compared with the other two isomers. This shallow HO state is ascribed to the highly distorted covalent bonds associated with the four-membered ring, leading to the higher total energy and the small HO-LU energy gap.

Figure 3 shows the squared wave functions of the HO and LU states of the three dicoronylene isomers. Interestingly, in all cases, the HO and LU states respectively exhibit antibonding and bonding nature with respect to the intermolecular covalent bonds. In the case of the isomer with the six-membered ring, the wave function distribution of 
(a)

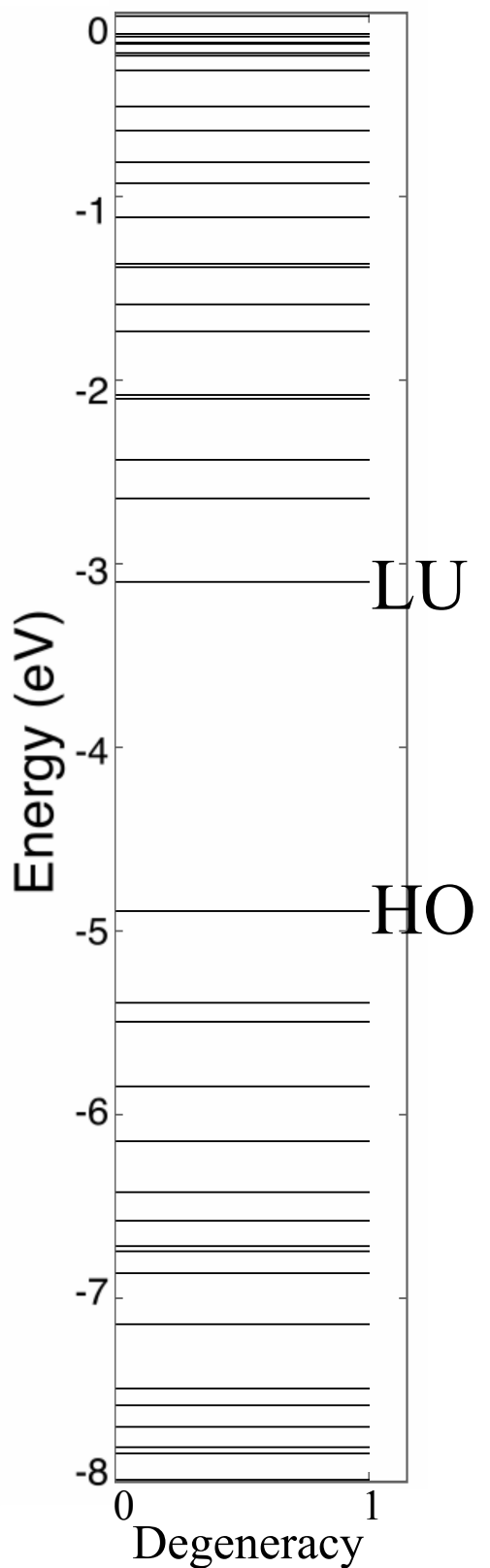

(b)

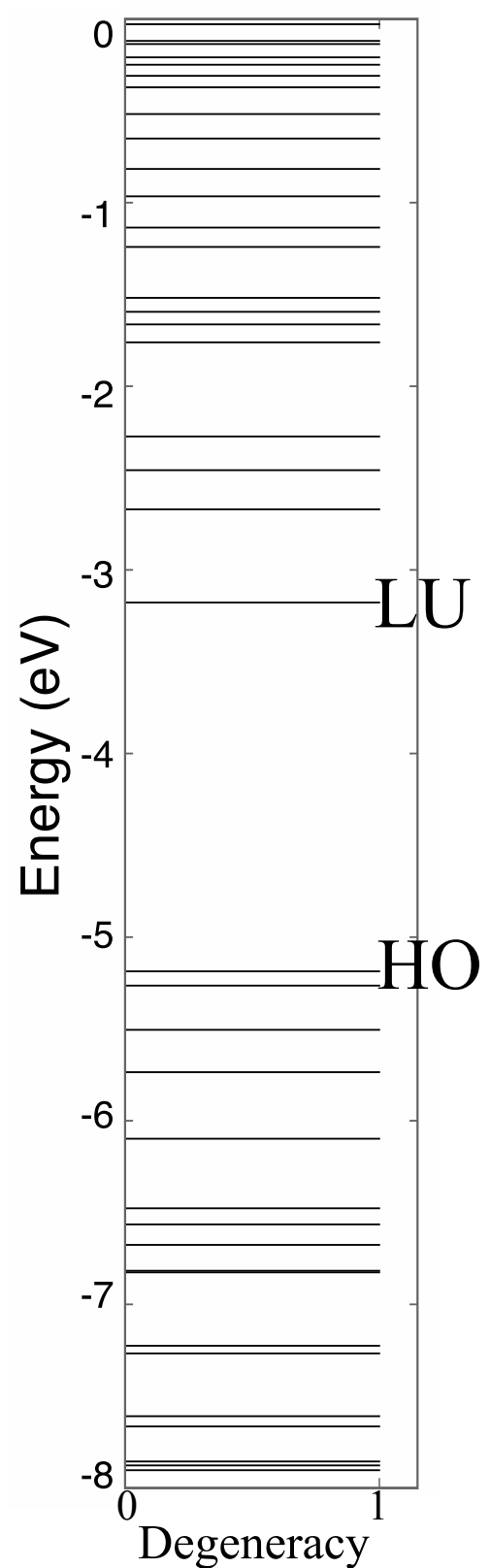

(c)

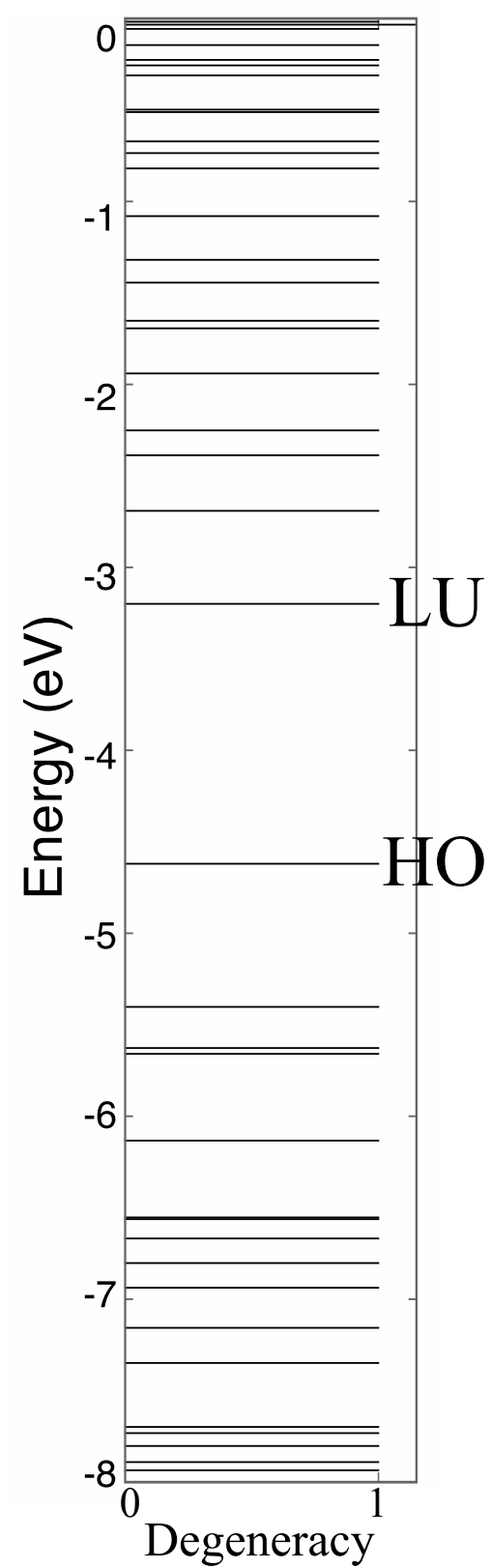

Fig. 2. Electronic structure of dicoronylenes molecules for isomers with (a) six-membered, (b) five-membered, and (c) four-membered rings. Energies are measured from that of the vacuum level.

both the HO and LU states is the same as that of the isolated coronene monomer, indicating that the dimerization does not affect the electronic structure of the dicoronylene with the six-membered intermolecular ring. For the other two isomers, the dimerization causes the modulation of the wave function distribution of the $\mathrm{HO}$ or LU states of the isolated coronene molecule. The LU state exhibits a similar distribution to that of the 
(a)
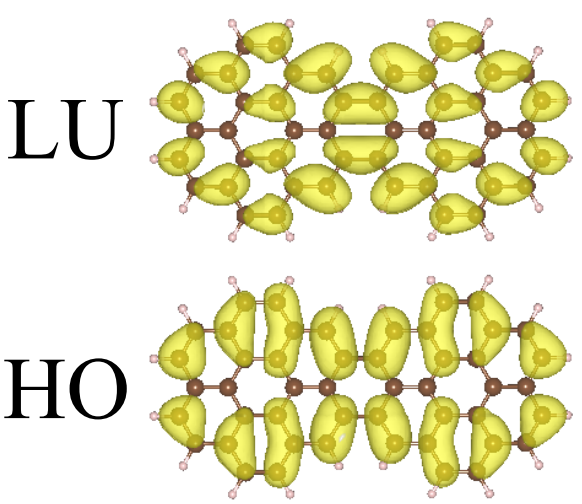

(b)
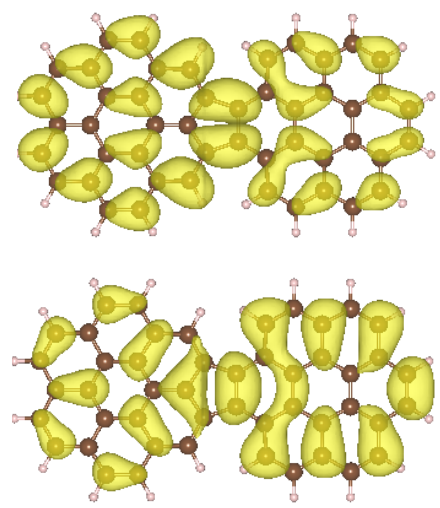

(c)
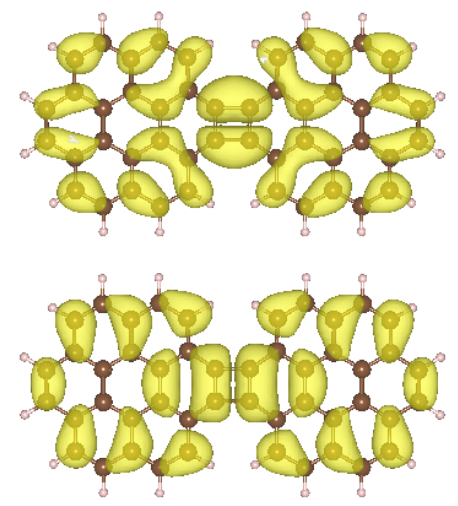

Fig. 3. (Color online) Squared wave functions of the $\mathrm{HO}$ and LU states of dicoronylene isomers with (a) six-membered, (b) five-membered, and (c) four-membered rings.

monomer while the $\mathrm{HO}$ state does not retain the distribution of the monomer for the isomer with the five-membered ring. The HO and LU states do not exhibit similar characteristics to those of the monomer for the isomer with the four-membered ring due to the substantially distorted covalent bonds between coronene units.

Figure 4 shows the optimized geometries of coronene polymers with four-membered, five-membered, and six-membered rings in the inter-coronene covalent bonds. The optimum inter-coronene distances are $8.52,8.57$, and $8.76 \AA$ for the polymers with sixmembered, five-membered, and four-membered rings, respectively. For the polymer with four-membered rings, the elongated intermolecular distance is ascribed to the substantial deformation of the coronene unit in accordance with the formation of four-membered rings. Indeed, the calculated bond lengths parallel to the polymer direction, $\mathrm{d} 1$, are elongated by about $0.1 \AA$ (Table III).

Table III. Bond lengths of coronene polymers (in A). Bond indexes are listed in Fig. 4.

\begin{tabular}{cccccccc}
\hline & $\mathrm{d} 1$ & $\mathrm{~d} 2$ & $\mathrm{~d} 3$ & $\mathrm{~d} 4$ & $\mathrm{~d} 5$ & $\mathrm{~d} 6$ & $\mathrm{~d} 7$ \\
\hline Polymer [6] & 1.45 & 1.42 & 1.38 & 1.41 & 1.41 & 1.4 & \\
Polymer [5] & 1.41 & 1.46 & 1.41 & 1.41 & 1.42 & 1.42 & 1.42 \\
Polymer [4] & 1.50 & 1.40 & 1.38 & 1.44 & 1.41 & 1.42 & \\
\hline
\end{tabular}

The total energy and Young's modulus of the coronene polymers are summarized in Table IV. The polymer with six-membered rings has a lower total energy than the poly- 
(a)

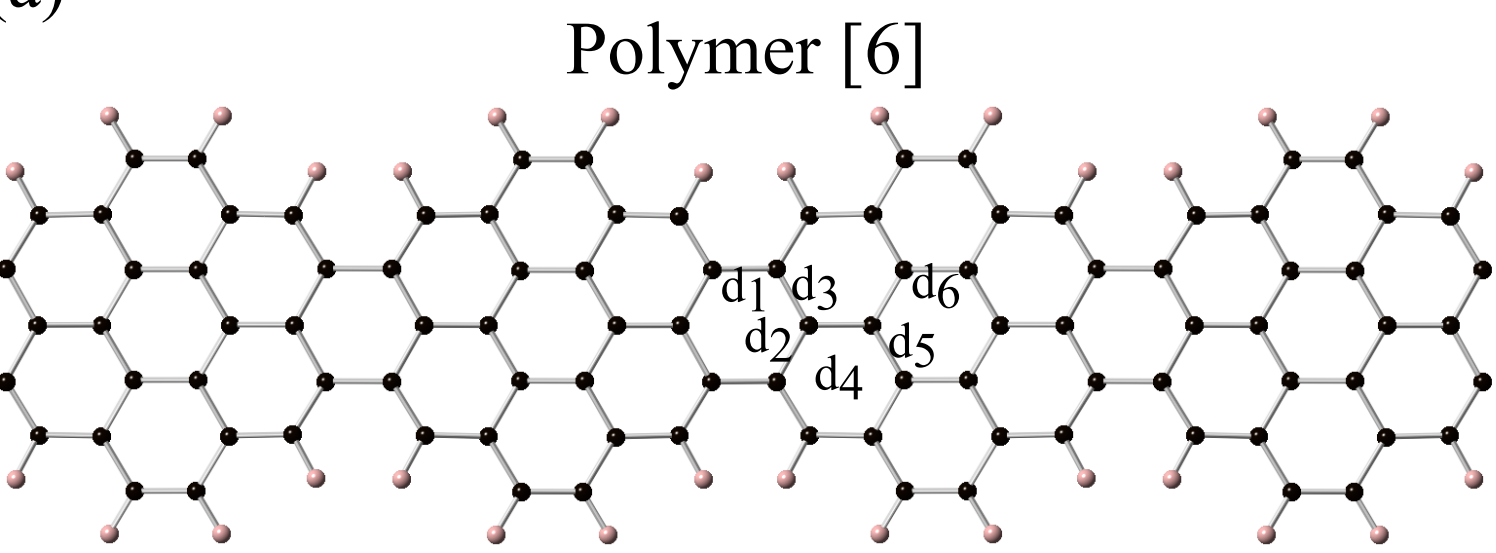

(b)

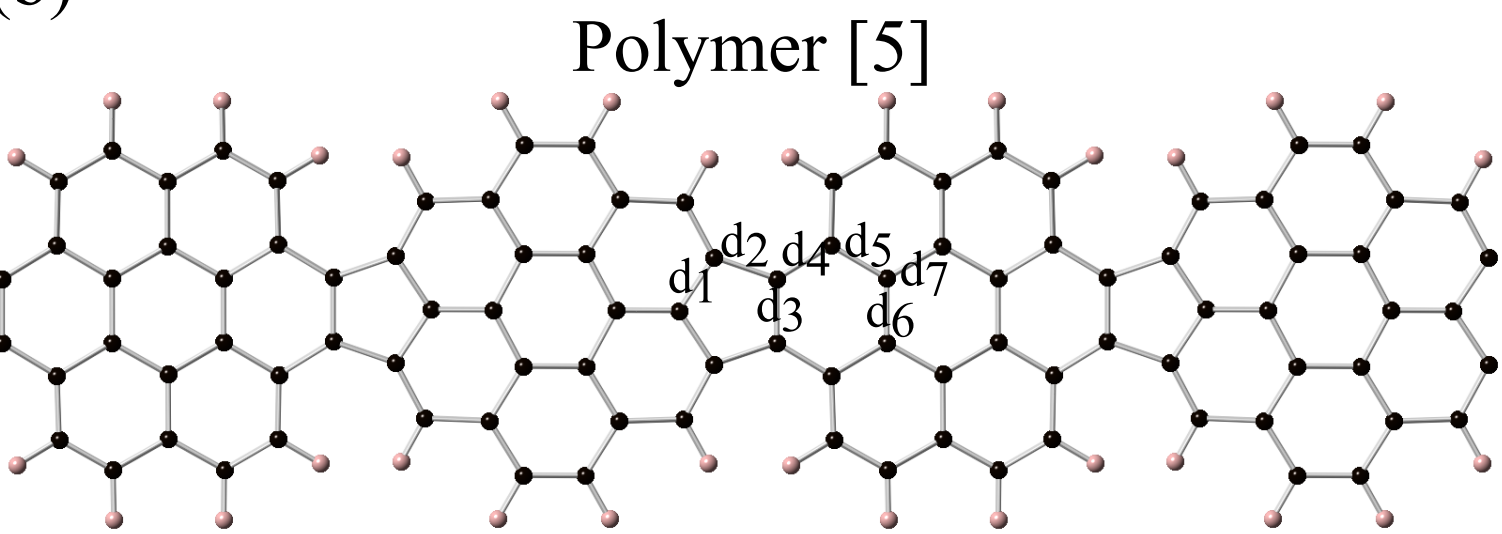

(c)

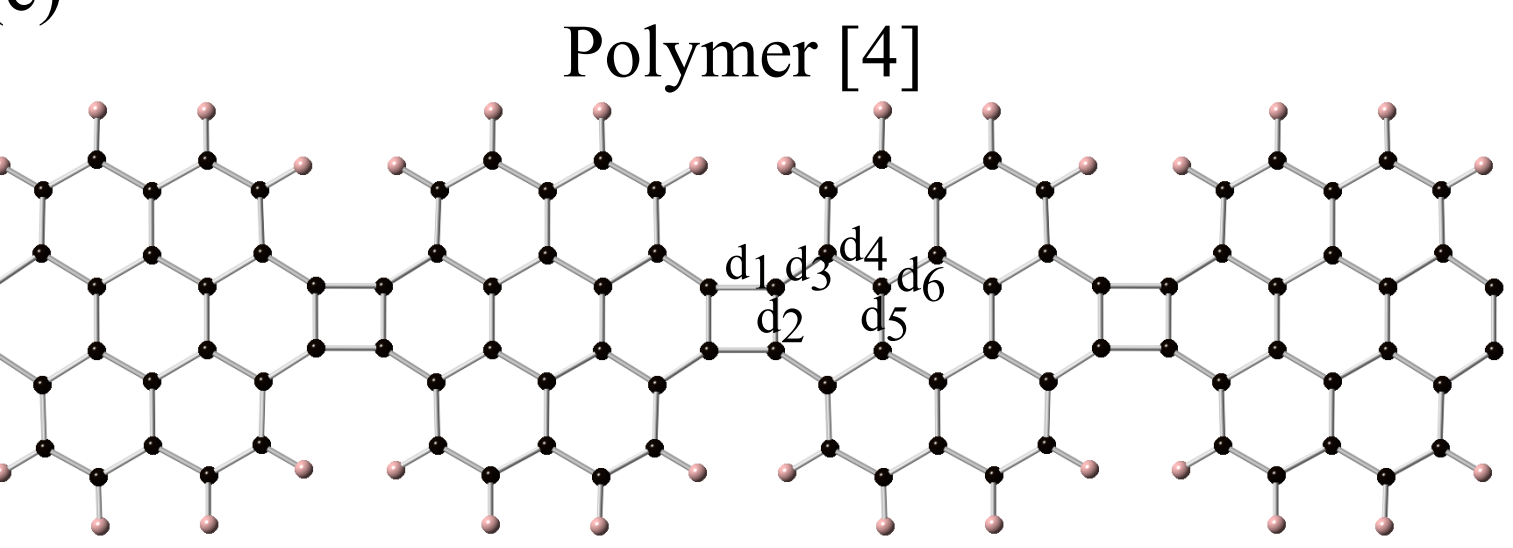

Fig. 4. Geometric structures of coronene polymers with (a) six-membered, (b) five-membered, and (c) four-membered rings.

mers with five-membered and four-membered rings by 0.57 and $2.51 \mathrm{eV}$ per coronene molecule, respectively. Thus, the polymer with six-membered intermolecular rings is the most stable among the three polymers studied here. Furthermore, as in the case 
Table IV. Relative total energies of coronene polymers. Energies are measured from that of the ground-state polymer containing six-membered rings between coronene molecules.

\begin{tabular}{lcc}
\hline & Energy (eV/coronene) & Young's modulus (GPa) \\
\hline Polymer [6] & 0 & 619 \\
Polymer [5] & 0.57 & 624 \\
Polymer [4] & 2.51 & 527 \\
\hline
\end{tabular}

of dicoronylene molecules, the polymer with five-membered rings has a slightly higher total energy than the polymer with six-membered rings, indicating that the polymer is also stable under ambient conditions. We also calculated the Young's modulus of the coronene polymers. To calculate the Young's modulus, we chose $3.35 \AA$ as the thickness of the polymers, corresponding to the van der Waals thickness of graphitic materials as used in previous works. ${ }^{29,30)}$ As shown in Table IV, the coronene polymers exhibit moderate stiffness with Young's moduli of 619,624, and $527 \mathrm{GPa}$ for the polymers with six-membered, five-membered, and four-membered rings, respectively, although some of these polymers have distorted $\mathrm{C}-\mathrm{C}$ bonds.

Figure 5 shows the electronic energy bands of the coronene polymers. All polymers are semiconductors with energy gaps of $0.84,1.56$, and $0.84 \mathrm{eV}$ for the polymers with six-membered, five-membered, and four-membered rings, respectively. The polymers with six-membered and four-membered rings possess a direct band gap at the $\Gamma$ point, while the polymer with five-membered rings possesses an indirect gap between the $\Gamma$ and $\mathrm{X}$ points for the bottom of the conduction band and the top of the valence band. Further investigation on the electronic energy bands of these polymers was carried out to clarifyunravel the unusual nature of their valence electron states: the band dispersion of several states is narrower than that of graphene nanoribbons without edge roughness. In particular, we found that all the electronic states of the polymer with five-membered rings exhibit a considerably small dispersion with a width of less than $1 \mathrm{eV}$, reflecting the peculiar bond arrangement between adjacent coronene molecules: one of the two coronenes has armchair edges while the other has zigzag edges. These small band dispersions are attributed to the edge roughness reflecting the shape of the coronene molecule, leading to the localization of particular electron states on each molecule.

Figure 6 shows the squared wave functions of the $\mathrm{HO}$ and LU states of the coronene polymers. In all cases, the intermolecular covalent bonds exhibit antibonding and bond- 
(a)

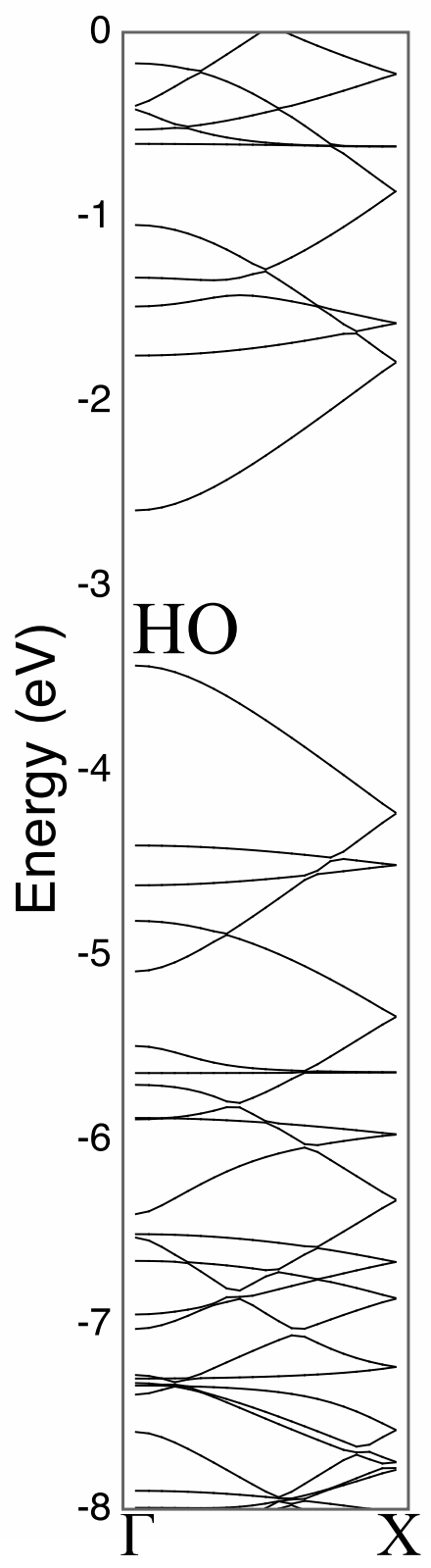

(b)

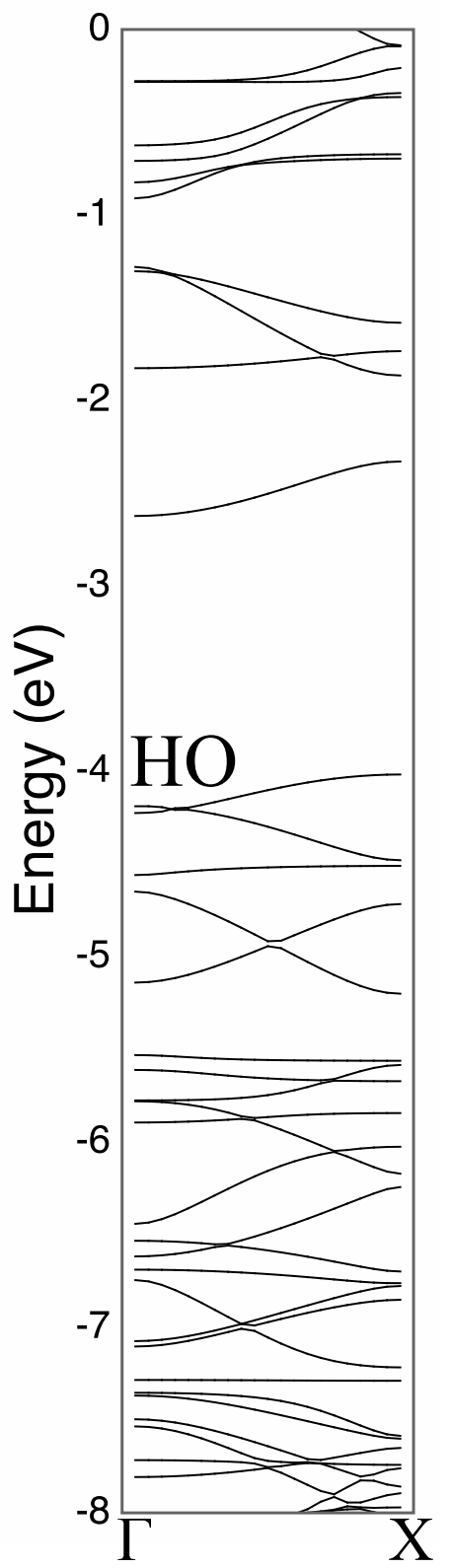

(c)

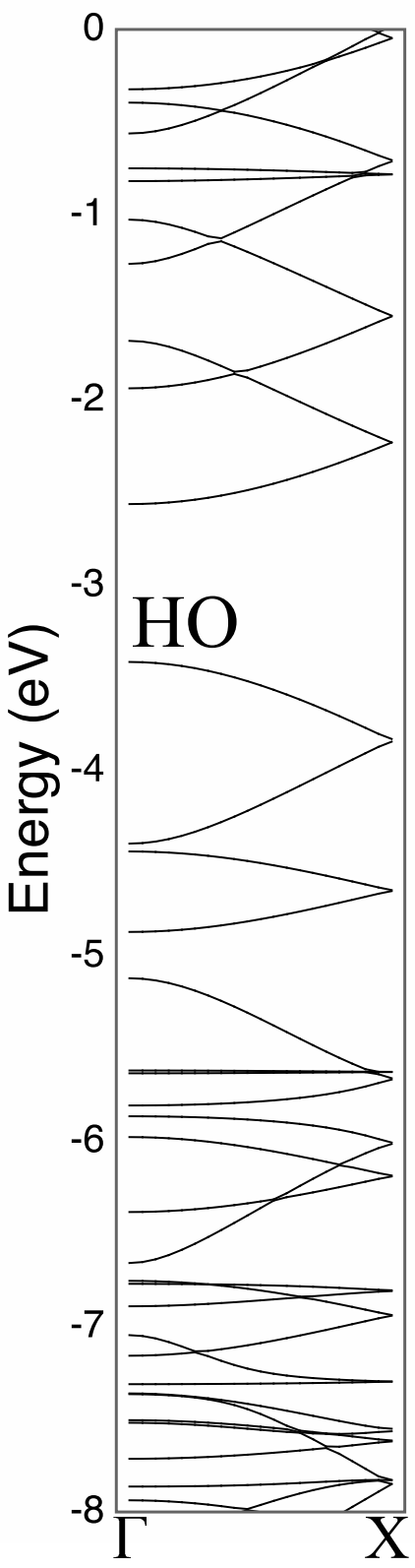

Fig. 5. Electronic energy bands of coronene polymers with (a) six-membered, (b) five-membered, and (c) four-membered rings. HO denotes the highest branch of the valence band. Energies are measured from that of the vacuum level.

ing $\pi$ natures for the $\mathrm{HO}$ and LU states, respectively. As in the case of dicoronylene molecules, the $\mathrm{HO}$ and LU states for the polymer with six-membered rings retain the $\mathrm{HO}$ and LU states of the isolated coronene molecule. For the polymer with five-membered rings, only the $\mathrm{HO}$ state retains the $\mathrm{HO}$ nature of the isolated coronene. 
(a)

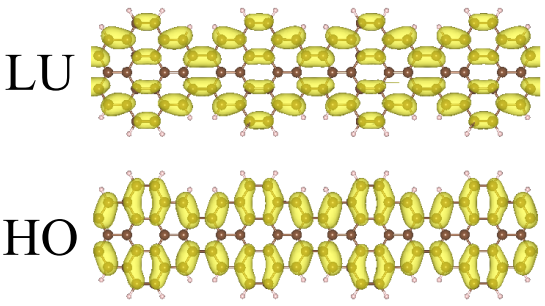

(b)

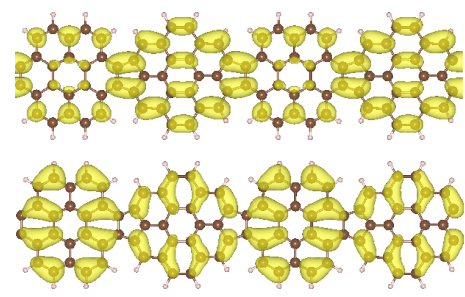

(c)

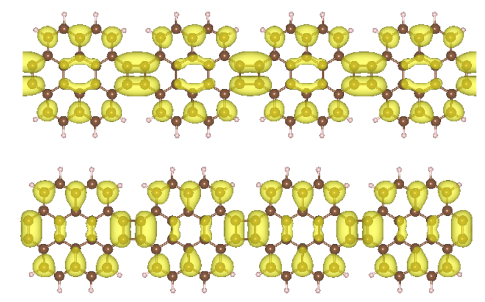

Fig. 6. Squared wave functions of $\mathrm{HO}$ and LU of coronene polymers with (a) six-membered, (b) five-membered, and (c) four-membered rings.

\section{Conclusions}

We studied the geometric and electronic structures of dimerized and one-dimensionally polymerized coronene molecules by first-principles total-energy calculation within the framework of DFT. Our calculations show that two of the dicoronylene molecules, which have been experimentally synthesized, have a relatively small total energy. While the remaining isomer has a higher total energy than these isomers by about $1 \mathrm{eV}$ per coronene molecule, ascribed to the formation of four-membered rings between the coronene molecules. Cyclodehydrogenation led to a decrease in the energy gap between the highest occupied and lowest unoccupied states of dicoronylene compared with that of an isolated coronene molecule. In the case of polymerized coronene, we found that the polymer with six-membered rings is the most stable of the isomers studied here. In addition, we also found that the total energy of the polymer with five-membered rings is slightly higher than that of the ground-state structure by $0.57 \mathrm{eV}$ per coronene. On the other hand, the remaining polymer containing four-membered rings is the least stable, whose energy is $2.5 \mathrm{eV}$ higher than that of the polymer with six-membered rings. These polymers possess moderate mechanical stiffness with a Young's modulus of about 600 GPa. All polymers are semiconductors with direct and indirect energy gaps of about 0.8 and $1.5 \mathrm{eV}$, respectively, depending on the mutual arrangement of the coronene molecules. Thus, by controlling the cyclodehydrogenation between coronene molecules, we can tailor the electronic properties of polymerized coronene for their use in a wide range of modern technologies.

\section{Acknowledgments}

This work was supported in part by a Grant-in-Aid for Scientific Research from the Ministry of Education, Culture, Sports, Science and Technology of Japan and the Joint 
Research Program on Zero-Emission Energy Research, Institute of Advanced Energy, Kyoto University. Computations were performed on an NEC SX-8/4B at the University of Tsukuba, an SGI ICE XA/UV at the Institute for Solid State Physics, The University of Tokyo, and an NEC SX-Ace at the Cybermedia Center, Osaka University. 


\section{References}

1) T. Seyller, K. Emtsev, F. Speck, K. Gao, L. Ley, A. Tadich, L. Broekman, J. D. Riley, R. C. G. Leckey, O. Rader, A. Varykhalov, and A. M. Shikin, Surf. Sci. 600, 3906 (2006).

2) K. S. Novoselov, A. K. Geim, S. V. Morozov, D. Jiang, M. I. Katsnelson, I. V. Grigorieva, S. V. Dubonos, and A. A. Firsov, Nature 438, 197 (2005).

3) Y. Zhang, Y. -W. Tan, H. L. Stormer, and P. Kim, Nature 438, 201 (2005).

4) X. Du, I. Skachko, F. Duerr, A. Luican, and E. Y. Andrei, Nature 462, 192 (2009).

5) K. I. Bolotin, F. Ghahari, M. D. Shulman, H. L. Stormer, and P. Kim, Nature 462, 196 (2009).

6) K. S. Novoselov, Z. Jiang, Y. Zhang, S. V. Morozov, H. L. Stormer, U. Zeitler, J. C. Maan, G. S. Boebinger, P. Kim, and A. K. Geim, Science 315, 1379 (2007).

7) K. S. Novoselov, E. McCann, S. V. Morozov, V. I. Fal'ko, M. I. Katsnelson, U. Zeitler, D. Jiang, F. Schedin, and A. K. Geim, Nat. Phys. 2, 177 (2006).

8) S. V. Morozov, K. S. Novoselov, and A. K. Geim, Phys. Rev. Lett. 97, 016801 (2006).

9) M. I. Katsnelson, K. S. Novoselov, and A. K. Geim, Nat. Phys. 2, 620 (2006).

10) M. S. Dresselhaus and G. Dresselhaus, Adv. Phys. 30, 139 (1981).

11) M. S. Dresselhaus and G. Dresselhaus, Adv. Phys. 51, 1 (2002).

12) A. H. C. Neto, F. Guinea, N. M. R. Peres, K. S. Novoselov, and A. K. Geim, Rev. Mod. Phys. 81, 109 (2009).

13) A. K. Geim and K. S. Novoselov, Nat. Mat. 6, 183 (2007).

14) S. Cho, Y. -F. Chen, and M. S. Fuhrer, Appl. Phys. Lett. 91, 123105 (2007).

15) M. Fujita, K. Wakabayashi, K. Nakada, and K. Kusakabe, J. Phys. Soc. Jpn. 65, 1920 (1996).

16) K. Nakada, M. Fujita, G. Dresselhaus, and M. S. Dresselhaus, Phys. Rev. B 54, 17954 (1996).

17) J. Wu, W. Pisula, and K. Müllen, Chem. Rev. 107, 718 (2007).

18) K. Müllen and J. P. Rabe, Acc. Chem. Res. 41, 511 (2008).

19) J. M. Robertson and J. G. White, J. Chem. Soc. 607 (1945).

20) J. K. Fawcett and J. Trotter, Proc. R. Soc. London, Ser. A 289, 366 (1966).

21) M. Fujihara, Y. Miyata, R. Kitaura, Y. Nishimura, C. Camacho, S. Irle, Y. Iizumi, T. Okazaki, and H. Shinohara, J. Phys. Chem. C 116, 15141 (2012). 
22) H. E. Lim, Y. Miyata, M. Fujihara, S. Okada, Z. Liu, Arifin, K. Sato, H. Omachi, R. Kitaura, S. Irle, K. Suenaga, and H. Shinohara, ACS Nano 9, 5034 (2015).

23) P. Hohenberg and W. Kohn, Phys. Rev. 136, B864 (1964).

24) W. Kohn and L. J. Sham, Phys. Rev. 140, A1133 (1965).

25) Y. Morikawa, K. Iwata, and K. Terakura, Appl. Surf. Sci. 169-170, 11 (2001).

26) J. P. Perdew, K. Burke, and M. Ernzerhof, Phys. Rev. Lett. 77, 3865 (1996).

27) M. Zander and W. Franke. Chem. Ber. 91, 2794 (1958) in German.

28) H. J. Lempka, S. Obenland, and W. Schmidt, Chem. Phys. 96, 349 (1985).

29) J. W. Jiang, J. S. Wang, and B. Li, Phys. Rev. B 80, 113405 (2009).

30) C. G. Lee, X. D. Wei, J. W. Kysar, and J. Hone, Science 321, 385 (2008). 